\title{
Editorial
}

\section{Aiming Too Low: Reevaluation of Target Concentrations of Serum 25-Hydroxyvitamin D in Secondary Hyperparathyroidism}

\author{
Anders H. Berg ${ }^{a}$ Ravi I. Thadhanib \\ a Departments of Pathology and Biomedical Sciences, Cedars-Sinai Medical Center, Los Angeles, CA, USA; \\ ${ }^{b}$ Departments of Medicine and Biomedical Sciences, Cedars-Sinai Medical Center, Los Angeles, CA, USA
}

What are the ideal concentrations of serum 25-hydroxyvitamin $\mathrm{D}(25 \mathrm{D})$ needed to maintain adequate amounts of active 1,25D-dihydroxyvitamin D $(1,25 \mathrm{D})$ and prevent maladaptive secondary hyperparathyroidism (SHPT) in patients with stage 3-4 chronic kidney disease (CKD)? Additionally, when treating SHPT with vitamin D supplements, what are the optimal concentrations of parathyroid hormone $(\mathrm{PTH})$ that one should target? Is SHPT associated with CKD always maladaptive, or is it an appropriate physiologic response to maintain calcium/phosphate homeostasis necessary to prevent adynamic bone disease? These are some important matters in question according to the intriguing new report by Strugnell et al. [1] published in this issue. To appreciate the significance of their findings in a historical context, we must first consider the evolution of consensus recommendations for treatment of SHPT in CKD.

In 2003, the Kidney Disease Outcomes Quality Initiative (KDOQI) group published guidelines recommending that patients with stage 3-5 CKD not on dialysis be monitored for SHPT and vitamin D deficiency. KDOQI's guidelines recommended therapy with ergocalciferol for patients with $25 \mathrm{D}$ levels below $30 \mathrm{ng} / \mathrm{mL}$ to achieve $25 \mathrm{D}$ levels $>30 \mathrm{ng} / \mathrm{mL}$, and active 1,25D therapy for patients with PTH values $>300 \mathrm{pg} / \mathrm{mL}$ irrespective of $25 \mathrm{D}$ levels

\section{KARGER}

(c) 2019 S. Karger AG, Basel

E-Mail karger@karger.com

www.karger.com/ajn
[2]. In 2009, the Kidney Disease Improving Global Outcomes group (KDIGO) modified these recommendations, suggesting that patients with CKD stages 3-5 with vitamin $\mathrm{D}$ deficiency or insufficiency be treated with natural vitamin D supplementation (ergocalciferol or cholecalciferol), but because of ongoing debate, the guideline did not specify thresholds for vitamin D deficiency or insufficiency [3]. At the same time, various authorities were defining vitamin $\mathrm{D}$ deficiency as concentrations of $25 \mathrm{D}<10-20 \mathrm{ng} / \mathrm{mL}$ and insufficiency as concentrations $<30-35 \mathrm{ng} / \mathrm{mL}$ [4]. The KDIGO group acknowledged that optimal concentrations of PTH in non-dialyzed CKD patients were not precisely known. Nevertheless, they recommended that patients with vitamin D insufficiency and PTH levels above the upper limit of normal should first be treated with the same cholecalciferol or ergocalciferol supplementation strategies used in the general population, and only when these natural vitamin D supplements failed to control increasing or persistently elevated $\mathrm{PTH}$, treatment with calcitriol or other active vitamin D analogs should be initiated. In 2017, KDIGO issued updated guidelines that remained unchanged except for the stipulation that calcitriol or active vitamin $\mathrm{D}$ analogs should not be routinely prescribed for stage 3-5 CKD adult patients not on dialysis, but 
should be reserved for stage 4-5 CKD patients with "severe and progressive SHPT" [5]. Their rationale cited recent randomized controlled trials (RCTs) that not only observed no measurable clinical benefits of these therapies but also revealed an increased risk of hypercalcemia $[6,7]$. It is noteworthy that KDOQI and KDIGO issued somewhat different recommendations for children with $\mathrm{CKD}$ and diabetic patients with stage $5 \mathrm{CKD}$, and the updated guidelines now recommend careful monitoring of renal function, hypercalcemia, and hyperphosphatemia to preempt the potential adverse effects of vitamin D supplementation. But do these guidelines work in practice for our patients? Further, does the introduction of an extended release calcifediol (ERC) therapy change the options for these patients?

In their new paper, Strugnell and colleagues report results of an RCT of non-dialyzed adult patients with stage 3 or 4 CKD treated for 26 weeks with ERC or placebo. Only patients with baseline 25D levels between 10 and 30 $\mathrm{ng} / \mathrm{mL}$ and HPT were included; those with hypercalcemia, hyperphosphatemia, nephrotic range proteinuria, or abnormally high urinary calcium excretion were excluded. Patients were monitored for changes in biomarkers of calcium homeostasis and bone mineral turnover. Their analysis stratified patients' results into quintiles according to the final concentrations of $25 \mathrm{D}$ achieved after supplementation and compared differences in average concentrations of biomarkers of calcium homeostasis and bone mineralization. The investigators found that ERC treatment successfully increased concentrations of $1,25 \mathrm{D}$ and lowered plasma PTH levels in a manner proportional to achieved levels of 25D. Furthermore, the authors found that PTH levels were suppressed significantly only in the group that achieved average concentrations of 25D of $50.8 \mathrm{ng} / \mathrm{mL}$ or greater. The authors also observed that increasing patients' $25 \mathrm{D}$ was associated with significant decreases in biomarkers of bone mineral turnover, thus corroborating that ERC treatment can successfully suppress the possibly maladaptive actions of PTH. Most importantly, in patients who achieved average concentrations of $25 \mathrm{D}$ as high as $92.5 \mathrm{ng} / \mathrm{mL}$, no significant changes in average serum calcium, serum phosphorous, eGFR, or urine calcium/creatinine ratio were observed, suggesting that even relatively high doses of ERC do not increase the risk of hypercalcemia or hyperphosphatemia.

Together these findings suggest that ERC may represent a new effective therapy for treatment of vitamin $\mathrm{D}$ deficient SHPT, and imply a need to reevaluate the currently recommended thresholds that define vitamin $\mathrm{D}$ sufficiency in CKD patients. In their study, only patients who achieved average 25D concentrations of $50.8 \mathrm{ng} / \mathrm{mL}$ responded with decreased PTH - a concentration that is dramatically higher than current therapeutic targets and the significance of this finding requires emphasis and explanation.

The most widely recognized and commonly cited clinical threshold for serum 25D "sufficiency" in the general population is $>30 \mathrm{ng} / \mathrm{mL}$. This value is based upon studies in which PTH levels were maximally suppressed by vitamin D supplementation, but none of these studies included patients with CKD [8]. Since the kidney plays an important role in vitamin $\mathrm{D}$ metabolism, a potentially serious confounding issue arises. CKD is associated with the upregulation ofFGF23 and suppression of $1 \alpha$-hydroxylase, which together result in decreased concentrations of 1,25D levels relative to 25D levels in CKD patients [9]. Furthermore, the choice to accept the same threshold to define 25D sufficiency in CKD patients as in the general population was based upon evidence that cholecalciferol or ergocalciferol therapies were minimally effective in ameliorating SHPT in patients with CKD [10]. The concentrations of 25D achieved using cholecalciferol in these RCTs were all lower than the levels achieved with the ERC regimen in the current study [11-13]. Since these earlier studies may not have achieved sufficient 25D levels, they would have been unlikely to detect a significant treatment effect on PTH. Together, these findings suggest that it may have been premature to conclude that the same threshold for 25D adequacy in CKD patients should be the same as for the general population.

Although the results in the current trial represent an enticing look into the effectiveness of a potential new tool for treatment of SHPT in CKD patients, the authors acknowledge that it remains unknown whether complete normalization of PTH is an appropriate goal for vitamin $\mathrm{D}$ repletion. This target may require higher doses of vitamin D supplementation that have the potential to pose increased risks of hypercalcemia, hyperphosphatemia, adynamic bone disease, or vascular calcification. Their study criteria included a precisely defined subset of CKD patients with no apparent deficits in calcium homeostasis, and as such, the safety of this treatment protocol cannot yet be generalized to include patients who do not fit into this specific profile. Their study size was also relatively modest and limited to 26 weeks of intervention. Nevertheless, these positive findings would lead us to conclude that larger and longer studies are worthy to pursue to further explore the safety and effectiveness of this potential new therapy for SHPT in CKD patients. 


\section{Disclosure Statement}

A.H.B. and R.I.T. are co-authors of patent WO2012094550A3 on measurement of Free or Bioavailable 25-hydroxyvitamin D. R.I.T. is a consultant to Fresenius Medical Care North America.

\section{Financial Support}

A.H.B. is supported by NIH grant R01 HL133399. R.I.T. is supported by NIH grant R01 HL117983.

\section{References}

1 Strugnell SA, Sprague SM, Ashfaq A, Petkovich M, Bishop CW. Rationale for Raising Current Clinical Practice Guideline Target for Serum 25-Hydroxyvitamin D in Chronic Kidney Disease. Am J Nephrol. 2019;49:284-293.

2 Hogg RJ, Furth S, Lemley KV, Portman R, Schwartz GJ, Coresh J, et al.; National Kidney Foundation's Kidney Disease Outcomes Quality Initiative. National Kidney Foundation's Kidney Disease Outcomes Quality Initiative clinical practice guidelines for chronic kidney disease in children and adolescents: evaluation, classification, and stratification. Pediatrics. 2003 Jun;111(6 Pt 1): 1416-21.

3 Kidney Disease: Improving Global Outcomes (KDIGO) CKD-MBD Work Group. KDIGO clinical practice guideline for the diagnosis, evaluation, prevention, and treatment of Chronic Kidney Disease-Mineral and Bone Disorder (CKD-MBD). Kidney Int Suppl. 2009 Aug;(113):S1-130.

4 Uhlig K, Berns JS, Kestenbaum B, Kumar R, Leonard MB, Martin KJ, et al. KDOQI US commentary on the 2009 KDIGO Clinical Practice Guideline for the Diagnosis, Evalua- tion, and Treatment of CKD-Mineral and Bone Disorder (CKD-MBD). Am J Kidney Dis. 2010 May;55(5):773-99.

5 Kidney Disease: Improving Global Outcomes (KDIGO) CKD-MBD Work Group. KDIGO 2017 Clinical Practice Guideline Update for the Diagnosis, Evaluation, Prevention, and Treatment of Chronic Kidney Disease-Mineral and Bone Disorder (CKD-MBD). Kidney Int Suppl (2011). 2017 Jul;7(1):1-59.

6 Wang AY, Fang F, Chan J, Wen YY, Qing S, Chan $\mathrm{IH}$, et al. Effect of paricalcitol on left ventricular mass and function in $\mathrm{CKD}-$ the OPERA trial. J Am Soc Nephrol. 2014 Jan; 25(1):175-86.

7 Thadhani R, Appelbaum E, Pritchett Y, Chang Y, Wenger J, Tamez H, et al. Vitamin $\mathrm{D}$ therapy and cardiac structure and function in patients with chronic kidney disease: the PRIMO randomized controlled trial. JAMA. 2012 Feb;307(7):674-84

8 Kramer H, Berns JS, Choi MJ, Martin K, Rocco MV. 25-Hydroxyvitamin D testing and supplementation in CKD: an NKF-KDOQI controversies report. Am J Kidney Dis. 2014 Oct;64(4):499-509.
9 Bosworth C, de Boer IH. Impaired vitamin D metabolism in CKD. Semin Nephrol. 2013 Mar;33(2):158-68.

10 Agarwal R, Georgianos PI. Con: nutritional vitamin $\mathrm{D}$ replacement in chronic kidney disease and end-stage renal disease. Nephrol Dial Transplant. 2016 May;31(5):706-13.

11 Dogan E, Erkoc R, Sayarlioglu H, Soyoral Y, Dulger $\mathrm{H}$. Effect of depot oral cholecalciferol treatment on secondary hyperparathyroidism in stage 3 and stage 4 chronic kidney diseases patients. Ren Fail. 2008;30(4):407-10.

12 Marckmann P, Agerskov H, Thineshkumar S, Bladbjerg EM, Sidelmann JJ, Jespersen J, et al. Randomized controlled trial of cholecalciferol supplementation in chronic kidney disease patients with hypovitaminosis D. Nephrol Dial Transplant. 2012 Sep;27(9): 3523-31.

13 Chandra P, Binongo JN, Ziegler TR, Schlanger LE, Wang W, Someren JT, et al. Cholecalciferol (vitamin D3) therapy and vitamin D insufficiency in patients with chronic kidney disease: a randomized controlled pilot study. Endocr Pract. 2008 Jan-Feb;14(1): 10-7. 